\title{
Dynamical Decoupling of Open Quantum Systems
}

\author{
Lorenza Viola ${ }^{1}$, Emanuel Knill ${ }^{2}$, and Seth Lloyd ${ }^{1} *$ \\ ${ }^{1}$ d'Arbeloff Laboratory for Information Systems and Technology, Department of Mechanical Engineering, \\ Massachusetts Institute of Technology, Cambridge, Massachusetts 02139 \\ ${ }^{2}$ Los Alamos National Laboratory, Los Alamos, New Mexico 87545
}

\begin{abstract}
We propose a novel dynamical method for beating decoherence and dissipation in open quantum systems. We demonstrate the possibility of filtering out the effects of unwanted (not necessarily known) system-environment interactions and show that the noise-suppression procedure can be combined with the capability of retaining control over the effective dynamical evolution of the open quantum system. Implications for quantum information processing are discussed.
\end{abstract}

03.65.-w, 03.67.-a, 05.30.-d

All real world quantum systems interact with their surrounding environment to a greater or lesser extent. Such systems are said to be open. No matter how weak the coupling that prevents the system from being isolated, the evolution of an open quantum system is eventually plagued by nonunitary features like decoherence and dissipation [1]. Quantum decoherence, in particular, is a purely quantum-mechanical effect whereby the system loses its ability to exhibit coherent behavior by getting entangled with the ambient degrees of freedom. Decoherence stands as a serious obstacle common to all applications relying on the capability of maintaining and exploiting quantum coherence. These encompass quantum state engineering [2], quantum interferometry [3], macroscopic quantum mechanics [4] and, notably, the whole emerging field of quantum information processing [5].

Recently, considerable effort has been devoted to design strategies able to counteract the effects of environmental couplings in open-system evolutions. In particular, the theory of quantum error correction has been developed to meet this challenge [6]. From a physical point of view, the general underlying question can be stated in terms of attaining quantum noise control. Unlike the closed-system limit, where the active manipulation of unitary dynamics is currently realized to be a problem of quantum control theory [7], the possibility of directly exploiting control techniques to influence opensystem properties has not been fully explored yet. Existing approaches mainly rely on feedback (or closed-loop) control configurations [8]. In fact, conventional quantum error correction protocols can be regarded, in their essence, as a form of quantum feedback control implemented on a redundant physical system.

In this Letter, we formulate a model for decoupling a generic open quantum system from any environmental interaction through simpler so-called open-loop control techniques. We show that the resulting description not only provides a comprehensive framework for decoherence-suppression schemes as first identified in [9] and subsequently implemented by various authors under specific assumptions [10], but, in contrast to previous proposals, it also points out a general criterion for engineering effective open-system evolutions that are, in principle, immune to noise and decoherence. More precisely, we find that one can effectively control the system to undergo a wide range of dynamical behavior while still eliminating the effects of the environment. The allowable dynamics are generated by a subgroup of the possible system tranformations. This has potentially important consequences for quantum control and quantum computation, in that it can be regarded as a strategy for performing fault-tolerant control. Even though the effects of the environment make it impossible to retain control over arbitrary unitary evolutions of a quantum system, an effective dynamics can be still reliably constructed over a restricted set of transformations.

The starting point of the method consists in recognizing that no relaxation process can take place instantaneously. Accordingly, one should be able to interfere with the associated dynamics by inducing motions into the systems, which are faster than the shortest time scale accessible to the reservoir degrees of freedom. The usage of tailored time-dependent perturbations as a tool to improve system performances has a long history within high-resolution Nuclear Magnetic Resonance spectroscopy, where versatile decoupling techniques are available to manipulate the overall spin Hamiltonian [11]. Despite this enlighting similarity, the construction of analogous procedures for open quantum systems faces an important conceptual difference, for we assume that any decoupling action can be exerted only on the system variables, the environment being contributed by a huge number of uncontrollable quantum degrees of freedom.

We consider a quantum system $S$ coupled to an arbitrary bath $B$, which together form a closed system defined on the Hilbert space $\mathcal{H}=\mathcal{H}_{S} \otimes \mathcal{H}_{B}, \mathcal{H}_{S}$ and $\mathcal{H}_{B}$

*Electronic addresses: vlorenza@mit.edu; knill@lanl.gov; slloyd@mit.edu. 
denoting $S$ and $B$ Hilbert spaces respectively. The overall Hamiltonian can be written in a concise form as

$$
H_{0}=H_{S} \otimes \mathbb{1}_{B}+\mathbb{1}_{S} \otimes H_{B}+H_{S B}=\sum_{\alpha} \mathcal{S}_{\alpha} \otimes \mathcal{B}_{\alpha},
$$

where $\mathbb{1}$ is the identity operator and the bath operators $\mathcal{B}_{\alpha}$ are supposed to be linearly independent. Being $H_{0}$ Hermitian, the linear space spanned by the system operators $\mathcal{S}_{\alpha}$ is a self-adjoint subspace in the vector space $B\left(\mathcal{H}_{S}\right)$ of bounded operators acting on $\mathcal{H}_{S}$. We shall assume that the unwanted noise-inducing Hamiltonian $H_{S B}$ is only contributed by a finite subset of open-system couplings. We call interaction space $\mathcal{I}_{S} \subseteq B\left(\mathcal{H}_{S}\right)$ the corresponding finite dimensional subspace. The second ingredient we introduce is the control algebra, $\mathcal{C}_{S}$, which is generated by the repertoire of Hamiltonians we can turn on for $S$ to implement decoupling. We allow for possibilities where $\mathcal{I}_{S} \neq \mathcal{C}_{S}$.

If $\rho_{\text {tot }}(0)=\rho_{S}(0) \otimes \rho_{B}(0)$ is the initial state over $\mathcal{H}$, the open-system evolution of $S$ is the coarse-grained dynamics $\rho_{S}(0) \mapsto \rho_{S}(t)=\operatorname{Tr}_{B}\left\{\rho_{\text {tot }}(t)\right\}, \operatorname{Tr}_{B}$ denoting partial trace over $\mathcal{H}_{B}$ [1]. The relaxation dynamics for $\rho_{S}(t)$, which involves a combination of quantum decoherence and dissipation mechanisms depending on the nature of the coupling operators, may display a complicate time dependence. In the simplest case, the off-diagonal matrix elements of $\rho_{S}(t)$ behave like $\exp \left(-t / \tau_{r e l}\right), \tau_{r e l}$ indicating the time scale for significant departure from unitarity and irreversible loss of quantum coherence.

In order to protect the evolution of $S$ against the effect of the interaction $H_{S B}$, we start by seeking a perturbation $H_{1}(t) \in \mathcal{C}_{S}$ to be added to $H_{0}$ as a suitable decoupling interaction, $H(t)=H_{0}+H_{1}(t) \otimes \mathbb{1}_{B}$. We restrict here to situations where the control field is cyclic, i.e., associated to a decoupling operator $U_{1}(t)$ that is periodic over some cycle time $T_{c}>0$ :

$$
U_{1}(t) \equiv T \exp \left\{-i \int_{0}^{t} d u H_{1}(u)\right\}=U_{1}\left(t+T_{c}\right) .
$$

In the interaction representation associated with $H_{1}(t)$, defined by $\rho_{\text {tot }}(t)=U_{1}(t) \tilde{\rho}_{t o t}(t) U_{1}^{\dagger}(t)$ on $\mathcal{H}$, time evolution is ruled by a transformed time-varying Hamiltonian,

$$
\tilde{H}(t)=U_{1}^{\dagger}(t) H_{0} U_{1}(t)=\sum_{\alpha}\left[U_{1}^{\dagger}(t) \mathcal{S}_{\alpha} U_{1}(t)\right] \otimes \mathcal{B}_{\alpha} .
$$

Since $U_{1}\left(T_{c}\right)=\mathbb{1}_{S}$, the evolution in the original Schrödinger representation can be easily derived. One can prove that after $N$ cycles, $T_{N}=N T_{c}$,

$$
U_{t o t}\left(T_{N}\right)=\mathrm{e}^{-i \bar{H} T_{N}},
$$

where the motion of the system under the timedependent field $H_{1}(t)$ has been replaced by a stroboscopic development under an effective so-called average Hamiltonian $\bar{H}$ 11]. The calculation of $\bar{H}$ is performed on the basis of a standard Magnus expansion of the time-ordered exponential defining the cycle propagator $U_{t o t}\left(T_{c}\right)=\exp \left(-i \bar{H} T_{c}\right)$,

$$
T \exp \left\{-i \int_{0}^{T_{c}} d u \tilde{H}(u)\right\}=\mathrm{e}^{-i\left[\bar{H}^{(0)}+\bar{H}^{(1)}+\ldots\right] T_{c}},
$$

where the various contributions collect terms of equal order in the transformed Hamiltonian. In particular,

$$
\bar{H}^{(0)}=\frac{1}{T_{c}} \int_{0}^{T_{c}} d u \tilde{H}(u) .
$$

We shall say that $k$ th-order decoupling is achieved if the control field $H_{1}(t)$ can be devised so that contributions mixing $S$ and $B$ degrees of freedom are no longer present in $\bar{H}^{(0)}$ and the first nonvanishing correction arises from $\bar{H}^{(k)}, k \geq 1$. Owing to the fact that the cycle time $T_{c}$ enters the Magnus series as a controllable expansion parameter, we examine the limit of fast control, where the lowest-order terms are expected to provide an accurate description (first-order decoupling). Formally, for a finite evolution time $T$, this requires considering $T_{c}=T / N$ in the limit as $N \rightarrow \infty$. The Magnus series defining evolution over a single cycle converges for sufficiently large $N$ whenever $\bar{H}^{(r)}=O\left(T_{c}^{r}\right)=O\left(1 / N^{2}\right)$ for $r \geq 2$. As a result, in the limit of arbitrarily fast control, contributions higher than zeroth-order are negligible in (5) and we can focus on the problem of designing the effective Hamiltonian $\bar{H}^{(0)}$.

We now show that the time average defining $\bar{H}^{(0)}$ can be made identical to a group-theoretical averaging procedure [12]. Since we can apply any Hamiltonian in the control algebra, full control of the decoupling propagator $U_{1}(t)$ is available over the associated set of unitary transformations. Let $\mathcal{G}$ be any finite group of unitary operators that generates $\mathcal{C}_{S}, \mathcal{G} \equiv\left\{g_{j}\right\}, j=0, \ldots,|\mathcal{G}|-1$, $|\mathcal{G}| \equiv \operatorname{ord}(\mathcal{G})$. Then the map

$$
\mathcal{S} \mapsto \overline{\mathcal{S}} \equiv \Pi_{\mathcal{C}}(\mathcal{S})=\frac{1}{|\mathcal{G}|} \sum_{g_{j} \in \mathcal{G}} g_{j}^{\dagger} \mathcal{S} g_{j}, \quad \mathcal{S} \in B\left(\mathcal{H}_{S}\right)
$$

is the unique operation projecting on the so-called centralizer of $\mathcal{G}$. Equivalently, since averaged operators $\overline{\mathcal{S}}$ commute with every $g_{j}$, they belong to the so-called commutant $\mathcal{C}$ of the control algebra. Notice that $\mathcal{C}$ is closed under commutation. The map (7) is implemented through a simple piecewise constant decoupling operator:

$$
U_{1}(t) \equiv g_{j}, \quad j \Delta t \leq t<(j+1) \Delta t,
$$

corresponding to a partition of the cycle time $T_{c}$ into $|\mathcal{G}|$ intervals of equal length $\Delta t \equiv T_{c} /|\mathcal{G}|$. Then, by (3),

$$
\bar{H}^{(0)}=\Pi_{\mathcal{C}}\left(H_{0}\right)=\sum_{\alpha} \overline{\mathcal{S}}_{\alpha} \otimes \mathcal{B}_{\alpha}
$$

which, by virtue of the quantum operation (7), displays well-defined symmetry properties. 
The decoupling prescription (8) requires the capability of instantaneously changing the evolution operator from $g_{j}$ to $g_{j+1}$ over successive subintervals, implying arbitrarily strong "kicks" of control field. Such impulsive full-power control configurations correspond to so-called quantum bang-bang controls as introduced in [9]. In fact, this method can be seen to provide an explicit control implementation of an abstract unitary symmetrization procedure recently proposed by Zanardi 13].

Two different situations arise depending on the knowledge available on the system-bath interaction $H_{S B}$. Let us first suppose that no knowledge is assumed, in which case the environmental coupling is completely arbitrary and $\mathcal{I}_{S}=B\left(\mathcal{H}_{S}\right)$. We can prove the following:

Theorem. Let $S$ be a finite dimensional system and let the interaction with the environment be arbitrary, $\mathcal{I}_{S}=B\left(\mathcal{H}_{S}\right)$. Then, in the limit of arbitrarily fast control rate, the evolution of observables in the control algebra can be suppressed arbitrarily well:

$$
\lim _{N \rightarrow \infty} \operatorname{Tr}_{S}\left\{A \rho_{S}\left(T=N T_{c}\right)\right\}=\operatorname{Tr}_{S}\left\{A \rho_{S}(0)\right\} .
$$

If, in addition, the control algebra is maximal, $\mathcal{C}_{S}=$ $B\left(\mathcal{H}_{S}\right)$, then complete first-order decoupling is achievable through system manipulations alone:

$$
\lim _{N \rightarrow \infty} \rho_{S}\left(T=N T_{c}\right)=\rho_{S}(0) .
$$

Proof. Let $A \in \mathcal{C}_{S}$. The first statement follows by Eq. (凷 with Hamiltonian (9) and the fact that $\left[A, \overline{\mathcal{S}}_{\alpha}\right]=0$ for every $\alpha$. If $\mathcal{C}_{S}$ consist of all operators, then the commutant $\mathcal{C}$ only contains $c$-numbers, $\overline{\mathcal{S}}_{\alpha}=\lambda_{\alpha} \mathbb{1}_{S}$ in (9) and the result follows.

The group able to average system operators into the commutant of $\mathcal{C}_{S}$ can be chosen as a set of linearly independent unitary operators realizing a so-called unitary error basis on $\mathcal{H}_{S}$ [14]. Such subgroups always exist for finite dimension. Within the class of piecewise constant decoupling sequences as considered above, it can then be shown that at least $\operatorname{dim}\left(\mathcal{C}_{S}\right)=|\mathcal{G}|$ steps are needed in a cycle to attain decoupling. Since the effective system evolution is completely quenched by the decoupling procedure, we call this configuration maximal averaging.

When some knowledge is available on the coupling $H_{S B}$, this information can be exploited to engineer shorter decoupling sequences fulfilling specific symmetry constraints. For a given (known) interaction space $\mathcal{I}_{S}$, the goal is to devise a control algebra able to selectively averaging out $\mathcal{I}_{S}, \Pi_{\mathcal{C}}\left(\mathcal{I}_{S}\right)=0$, while leaving invariant the sector in the operator space of the system containing some useful dynamics. This requires that the errorinducing and the desired system operators in (11) transform according to different irreducible representations of $\mathcal{G}$. By using (9), the effective open-system evolution over time $T$ is then governed by

$$
\lim _{N \rightarrow \infty} \rho_{S}\left(T=N T_{c}\right)=e^{-i \bar{H}_{S} T} \rho_{S}(0) e^{+i \bar{H}_{S} T},
$$

the projected Hamiltonian $\bar{H}_{S}=\Pi_{\mathcal{C}}\left(H_{S}\right)$ only consisting of operators belonging to $\mathcal{C}$. Equivalently, the set of operators in the commutant of $\mathcal{C}_{S}$ determines the reliable manipulations left available for effective system control. For a given $\mathcal{I}_{S}$, the identification of a minimal group $\mathcal{G}$ able to produce decoupling is nontrivial. We provide an illustrative example.

Let us discuss a $K$-qubits dissipative quantum register [5]. The maximum possible complexity of error generation arises in the presence of so-called total decoherence, whereby combined errors can occur to any number of qubits. In this case, $\mathcal{I}_{S}=B\left(\mathcal{H}_{S}\right) \simeq\left(\mathrm{C}^{2}\right)^{\otimes K}$ and maximal averaging is demanded to decouple the register from quantum noise. Since an error basis on $\mathrm{C}^{2 \otimes K}$ is generated via the tensor product of the standard Pauli bit/sign-flip error basis [14], a possible choice is $\mathcal{G}=\left\{\mathbb{1}_{S}, \sigma_{\alpha}^{(i)}\right\}^{\otimes K}, \alpha=x, y, z, i=1, \ldots, K$. Thus, a number of $|\mathcal{G}|=4^{K}$ steps is required for minimal length decoupling sequences. A more efficient averaging is possible if the relevant register-bath coupling is known to be linear in single-qubit operators, $H_{S B}=\sum_{i, \alpha} \sigma_{\alpha}^{(i)} \otimes \mathcal{B}_{\alpha}^{(i)}$. Under that condition, which is met for both independent decoherence $\left(\operatorname{dim}\left(\mathcal{I}_{S}\right)=3 K\right)$ and collective decoherence $\left(\operatorname{dim}\left(\mathcal{I}_{S}\right)=3\right)$, selective averaging suffices to decouple from errors. One can show that the tensor power of the Pauli group, $\mathcal{G}=\left\{\mathbb{1}_{S}, \otimes_{i=1}^{K} \sigma_{\alpha}^{(i)}\right\}, \alpha=x, y, z,|\mathcal{G}|=4$, represents a minimal choice. In terms of the control field $H_{1}(t)$, decoupling is then enacted by cycling the qubits in the register through sequences of collective $\pi$-pulses along two axes, e.g., $\Delta t-\pi_{x}-\Delta t-\pi_{-z}-\Delta t-\pi_{-x}-\Delta t-\pi_{-z}$, $\Delta t=T_{c} / 4$. In the special case of a purely decohering coupling, $H_{S B}=\sum_{i} \sigma_{z}^{(i)} \otimes \mathcal{B}_{z}^{(i)}$, the scheme can be further simplified by taking $\mathcal{G}=\left\{\mathbb{1}_{S}, \otimes_{i=1}^{K} \sigma_{x}^{(i)}\right\},|\mathcal{G}|=2$, which reproduces the situation analyzed in [9] for $K=1$. In the decoupling regime, the register effectively behaves as a noiseless quantum memory, which is an essential ingredient for various quantum cryptographic and communication schemes [5,15]. In addition, one can now perform in a fault-tolerant way any logical operation belonging to the commutant of the tensor power Pauli group, which is generated through commutation by matrices of the form $\sigma_{\alpha}^{(i)} \sigma_{\alpha}^{(j)}, i, j \in\{1, \ldots, K\}, \alpha=x, y, z$.

The results discussed so far show that decoherence and decay can be completely suppressed in the limit of infinitesimally short control time scale $\Delta t \rightarrow 0$. In order to convert this mathematical limit into a physically meaningful condition, we recall that complete information about the fluctuation-dissipation properties of a macroscopic bath is encapsulated in the spectral density function $J(\omega)$, measuring the density of modes at frequency $\omega$ multiplied by the square of the system-mode coupling strength. Quite generally, relaxation rates $\gamma=\tau_{r e l}^{-1}$ arise from an integration over the reservoir modes of the effects due to thermal and vacuum fluctuations, weighted with $J(\omega)$. This integration does not extend to arbitrarily large frequencies. For every physical spectral density 
function, a finite ultraviolet cut-off frequency $\omega_{c}$ always exists, such that $J(\omega) \rightarrow 0$ for $\omega>\omega_{c}$. The characteristic memory time $\tau_{c} \sim \omega_{c}^{-1}$, which is often set to be zero as a part of Markov approximation [1], determines the fastest time scale accessible to reservoir correlations. The condition $\Delta t \ll \tau_{c}$ replaces, in a realistic scenario, the ideal limit $\Delta t \rightarrow 0$. Thus, the physical requirement for decoupling is $\Delta t \lesssim \tau_{c}$ or $\omega_{c} \Delta t \lesssim 1$, i.e., the motion induced by the decoupling field needs to be faster than the fastest time scale characterizing the unwanted interactions.

In the presence of a small but finite decoupling time scale $\Delta t$, the representation of the average Hamiltonian $\bar{H}$ in terms of the lowest-order contribution $\bar{H}^{(0)}$ is approximate due to the higher-order terms. Consequently, decoupling is imperfect and relaxation dynamics still occurs even in the presence of control. For first-order decoupling, the leading correction is due to $\bar{H}^{(1)}$ and the key observation to estimate the decoupling accuracy as a function of $\Delta t$ is that the overall coupling strength to the bath has been renormalized by a factor of the order $\omega_{c} \Delta t$. Since the spectral density depends quadratically upon the interaction strength, all the relevant relaxation effects tend to be suppressed by a factor of the order $\left(\omega_{c} \Delta t\right)^{2}$. For a generic $k$ th-order decoupling scheme, the controlled relaxation rate is able to be reduced as

$$
\frac{\gamma^{C}}{\gamma} \approx\left(\omega_{c} \Delta t\right)^{2 k}=\left(\frac{\Delta t}{\tau_{c}}\right)^{2 k}, \quad k \geq 1
$$

Second-order decoupling can be realized via so-called symmetric cycles, whereby $U_{1}\left(T_{c}-t\right)=U_{1}(t)$. Being $\bar{H}^{(r)}=0$ for $r$ odd, the error is improved to $O\left(\Delta t / \tau_{c}\right)^{4}$. Iterative pulse sequences for $k$ th-order decoupling can be designed for specific systems like quantum registers.

In practice, the feasibility of the approach depends on both the relevant correlation times and the sophistication of the technology available to manipulate the specific physical system. In pulsed-NMR experiments [11], where relaxation mechanisms due to "slow" nuclear motions may involve correlation times longer than $10^{-8} \mathrm{~s}$, the main current limitation is represented by the pulse duration, $\tau_{P} \approx 1 \mu \mathrm{s}$. In atomic physics, decoupling methods could prove to be viable for damped harmonic oscillators schematizing the vibrational motion of trapped ions, since relevant cut-off frequencies may be estimated around $100 \mathrm{MHz}$ and a variety of experimental techniques exists for coherent optical manipulation [2]. As another potential area of applications, we mention semiconductor-based structures. Here, correlation times around $\omega_{\text {Debye }}^{-1} \approx 10^{-13} \mathrm{~s}$ are comparable to the sub-ps time scale where control operations have been demonstrated 16 and longer than the femtosecond scale of modern ultrafast laser technology. Rapid advancements in the capabilities of coherent control gives hope that, if not within the reach of present technology, implementations of decoupling schemes can be envisaged in a close future. In particular, since quantum computing resources are still a stringent practical requirement, decoupling techniques could be valuable compared to conventional error-correction networks in the field of NMR, ion-trap or solid state quantum computation.

In summary, we showed how to manipulate the irreversible component of open-system evolutions through the application of external controllable interactions. Maximal and selective decoupling were introduced within a common group-theoretical framework and their relevance to the issue of designing controlled effective opensystem evolutions elucidated. In the spirit of weakening the decoupling requirements as much as possible, the main question raised by the present analysis concerns the characterization of fault-tolerant decoupling schemes or, equivalently, the degree of decoupling attainable in the presence of power-limited and imperfect control operations. Work in ongoing along these directions.

This work was supported by ONR, AFOSR, and DARPA/ARO under the Quantum Information and Computation initiative and the NMR Quantum Computing initiative. E. K. received support from the NSA.

[1] C. W. Gardiner, Quantum Noise (Springer, Berlin, 1991).

[2] D. J. Wineland et al., Proc. Roy. Soc. London Ser. A 454, 411 (1998), and references therein.

[3] Quantum Interferometry, F. De Martini, G. Denardo, and Y. Shih Eds. (VCH Publishing Group, Berlin, 1996).

[4] Macroscopic Quantum Tunnelling and Coherence, special issue of J. Supercond., in press (October 1998).

[5] Quantum Communication, Computing, and Measurement, O. Hirota, A. S. Holevo, and C. M. Caves Eds. (Plenum Press, New York, 1997).

[6] (a) P. W. Shor, Phys. Rev. A 52, R2493 (1995); (b) A. M. Steane, Phys. Rev. Lett. 77, 793 (1996); (c) E. Knill and R. Laflamme, Phys. Rev. A 55, 900 (1997).

[7] A. G. Butkovskiy and Yu. I. Samoilenko, Control of Quantum Mechanical Processes and Systems (Kluwer Academic, Dordrecht, 1990).

[8] H. M. Wiseman and G. J. Milburn, Phys. Rev. Lett. 70, 548 (1993); D. Vitali, P. Tombesi, and G. J. Milburn, Phys. Rev. Lett. 79, 2442 (1997).

[9] L. Viola and S. Lloyd, Phys. Rev. A 58, 2733 (1998).

[10] M. Ban, J. Mod. Opt. 45, 2315 (1998); L. M. Duan and G. C. Guo, LANL e-print quant-ph/9807072; D. Vitali and P. Tombesi, LANL e-print quant-ph/9808055.

[11] R. R. Ernst, G. Bodenhausen, and A. Wokaun, Principles of Nuclear Magnetic Resonance in One and Two Dimensions (Clarendon Press, Oxford, 1987).

[12] J. F. Cornwell, Group theory in Physics, Vol. II (Academic Press, London, 1984).

[13] P. Zanardi, LANL e-print quant-ph/9809064.

[14] E. Knill, LANL e-print quant-ph/9608049.

[15] L. Goldenberg and L. Vaidman, Phys. Rev. Lett. 75, 1239 (1995).

[16] A. P. Heberle, J. J. Baumberg, and K. Kohler, Phys. Rev. 
Lett. 75, 2598 (1995). 